\title{
Medizin als Show
}

Es war ein medialer Coup der Sonderklasse, den die niederländische TV-Produktionsfirma «Endemol» vor einigen Wochen landete. Und zwar gleich ein doppelter: Zunächst lösten die cleveren Fernsehmacher mit der Ankündigung einer Organspendeshow, in der drei männliche Patienten um die Gunst und die Niere einer todkranken jungen Frau wetteifern sollten, einen weltweiten Protest-Tsunami aus. «Makaberes Spiel einer moralisch verkommenen Medienagentur» (Jörg-Dietrich Hoppe, Präsident der deutschen Bundesärztekammer gemäss Tagesspiegel online) war noch einer der gemässigteren Kommentare, die zum Vorhaben abgegeben wurden.

Als der Spuk vorbei war und sich alles als Bluff entpuppt hatte - die angeblich Todkranke war eine Schauspielerin, Organe wurden in der Sendung keine vergeben -, verblubberte die Empörungswelle rasch auf wundersame Weise. Ja, ein Grossteil der verschaukelten Öffentlichkeit, darunter auch Politiker, Transplantationsmediziner und Ethiker, zog unversehens eine positive Bilanz der Inszenierung. Natürlich, so der Tenor, hätten die Produzenten der Sendung zu ziemlich deftigen, mitunter auch bedenklichen Mitteln gegriffen, um auf ihr Anliegen, den Mangel an Spenderorganen, aufmerksam zu machen. Aber der niederländische Medienminister war bei weitem nicht der einzige, der plötzlich fand (ebenfalls laut Tagesspiegel online), die Show sei «eine intelligente Art», um für ein ernstes Problem zu sensibilisieren.

So, so - die Erfinder von Big Brother, bekannt für ihr soziales Engagement, wollten also sensibilisieren. Sie hatten eine Mission. Natürlich, der Gründer des Senders BNN, auf dem die Show zu sehen war, hatte selbst unter einer Nierenkrankheit gelitten und war letztlich daran gestorben. Je nach Quelle hatte er «nie eine Spenderniere erhalten» (z.B. T-online-Nachrichten) oder war fünf Jahre nach erfolgter Transplantation gestorben (z.B. News orf.at). Doch es wäre kleinlich, auf solchen Details herumzureiten.

Sogar ketzerisch wäre es wohl, anzunehmen, der Mechanismus könnte andersherum gespielt haben. Die Ausgangsfrage der TV-Produzenten hätte dann zum Beispiel gelautet: Mit welcher
Strategie erregen wir ein Höchstmass an Aufmerksamkeit, erzielen eine Traumquote, steigern damit unsern Marktwert und lassen so nebenbei ganz schön die Kasse klingeln? Zuerst als böse Buben dastehen, um sich dann - Tusch - unversehens in weisse Ritter im Dienst der guten Sache zu verwandeln. Wer kann schon etwas gegen ein Konzept sagen, das die Nachfrage nach Organspenderausweisen in verschiedenen Ländern sprunghaft in die Höhe schnellen lässt? Das Experten und anderen engagierten Persönlichkeiten Gelegenheit gibt, sich ernsthaft zu einem medizinischen Notstand zu äussern? Ziemlich genial, wie gesagt.

Jede Gesellschaft hat die Fernsehsendungen, die sie verdient, könnte man dazu auch feststellen. Medizin als Show - das gibt es in mehr oder weniger dezenter Form längst, auch hierzulande. Kranke betreten die Fernsehbühne schon fast wie Stars, getragen vom warmen Applaus des Studiopublikums: «Darf ich vorstellen, Herr X, er leidet an einer Herzinsuffizienz» - Applaus. «Frau Y, Fibromyalgie-Syndrom» - Applaus. «Herr Z. therapieresistente Depression»-Applaus.

An die intrinsische Verlogenheit solcher Formate hat man sich im Zeitalter von Talkshows zu Themen wie «Hilfe, mein Rehpinscher geht fremd» leider schon ziemlich gewöhnt. Zu hoffen, die Bilanz derartiger Sendungen könnte unter dem Strich aller Fragwürdigkeit zum Trotz positiv sein, scheint da reichlich optimistisch. Shows dieser Art werden als Instant-Unterhaltung konsumiert, die wohl nur in Ausnahmefällen mehr als einen kurzfristigen Betroffenheitsschauer erzeugt. Würde jede Woche eine Organspendeshow am Fernsehen gezeigt, sänke das Zuschauerinteresse sehr rasch, von der Quote derjenigen, die danach einen Spenderausweis bestellen, ganz zu schweigen.

Es bleibt also nur das stetige Drehen an der Sensationsschraube, und offensichtlich machen wir als Gesellschaft dieses Spiel ganz gerne mit. Bis zu welchem Punkt, wird sich zeigen. Vielleicht werden wir ja schon bald drei Lebensmüde im Studio sehen, die um einen assistierten Suizid im Luxushotel spielen.

Bruno Kesseli 